\title{
Cardio-cerebral infarction in left MCA strokes: a case series and literature review
}

\author{
Elochukwu Ibekwe $^{1}$ () $\cdot$ Hera A. Kamdar ${ }^{1} \cdot$ Tamara Strohm $^{1}$
}

Received: 25 May 2021 / Accepted: 23 September 2021 / Published online: 29 September 2021

(c) Fondazione Società Italiana di Neurologia 2021

\begin{abstract}
The objective of this manuscript is to describe the challenges of Cardio-Cerebral Infarction (CCI) treatment and to highlight the variable approaches in management. CCI is a rare clinical presentation of simultaneous acute ischemic stroke (AIS) and acute myocardial infarction (AMI) and poses a therapeutic challenge for practitioners. Each disease requires timely intervention to prevent irreversible damage; however, optimal management remains unclear. We describe three cases of CCI. All three patients presented with symptomatic left MCA (M1) occlusion, with ST elevation myocardial infarction (STEMI) and left ventricular apical thrombus. Fibrinolysis and mechanical thrombectomy (MT) were discussed in all cases, but only one patient received alteplase $(0.9 \mathrm{mg} / \mathrm{kg}$ ) and none underwent MT. Percutaneous intervention (PCI) was done in only one case. The two patients that did not receive thrombolysis were treated with modified therapeutic heparin (no bolus), and all received antiplatelet therapy. Ultimately, all three patients passed away. CCI poses a clinical challenge for physicians including (1) optimal strategies to enable swift mechanical reperfusion to both the brain and myocardium; (2) difference in dosage of thrombolytics for AIS versus AMI; (3) risk of symptomatic intracerebral hemorrhage following administration of anticoagulation and/or antiplatelet therapy; and (4) caution with use of thrombolytics in the setting of acute STEMI due to the risk of myocardial rupture. In the absence of high quality evidence and clinical guidelines, treatment of CCI is highly individualized.
\end{abstract}

Keywords Acute ischemic stroke $\cdot$ Cardio-cerebral infarct $\cdot$ Cerebral ischemia $\cdot$ Myocardial infarct $\cdot$ Alteplase

\section{Introduction}

Acute ischemic stroke (AIS) and acute myocardial ischemia (AMI) are the leading causes of morbidity and mortality globally [1]. Optimal management prioritizes revascularization via time-sensitive fibrinolysis, percutaneous intervention (PCI), mechanical thrombectomy (MT), and antiplatelet or anticoagulation therapy [2]. Simultaneous presentation of AIS and AMI within $48 \mathrm{~h}$, termed Cardio-Cerebral Infarction (CCI), is quite rare and may be associated with high

Elochukwu Ibekwe

ibek01@osumc.edu

Hera A. Kamdar

hera.kamdar@osumc.edu

Tamara Strohm

tamara.strohm@osumc.edu

1 Department of Neurology and Neurocritical Care, The Ohio State University, 395 West 12th Avenue, 7th Floor, Columbus, OH 43210, USA morbidity and mortality [3]. In patients with CCI, optimal administration of fibrinolytics (dose and agent), timing of intervention (PCI versus MT), treatment with anticoagulation and antiplatelet therapies, and rates of symptomatic ICH are unclear [4, 5]. Thus, optimal management is controversial, with added challenges in the event of a left ventricular (LV) thrombus.

Our objective is to review the epidemiology, pathophysiology, and management of CCI, along with the many management challenges.

\section{Methods}

This is a case series presenting observational data collected from three patients at The Ohio State University, Wexner Medical Center with acute presentation of CCI within a 1-month period in the year 2020. Due to the COVID-19 pandemic, verbal consent was obtained via telephone. 
Furthermore, a literature review of CCI was performed in PubMed and EMBASE. No time limitation filter was applied. The following MeSH terms were used as follows: "Cardio cerebral infarct," "stroke and MI," "stroke and myocardial infarction," "STEMI and stroke," "NSTEMI and stroke." Articles included, contained a full description of the case, outlining the presentation and management steps, and with evidence of both cerebral and cardiac infarction within $48 \mathrm{~h}$.

\section{Case summary}

We describe three cases of CCI in a 43-year-old male, a 72-year-old male, and an 80-year-old female (Table 1). All three patients presented with symptomatic proximal left MCA (M1) occlusion (Fig. 1), ST elevation myocardial infarction (STEMI) (Fig. 2), and left ventricular apical thrombus. Examinations revealed full left MCA syndrome with aphasia and R sided weakness (NIHSS 17-25). Interventional therapies such as alteplase and mechanical thrombectomy (MT) were discussed in all cases, but alteplase was utilized in only one. In one case, left heart catheterization was done without intervention/stenting. Two patients received modified therapeutic heparin (no bolus) for LV thrombus; all patients received antiplatelet therapy with aspirin and/or clopidogrel. Ultimately, all three patients expired.

\section{Cases}

\section{Case presentation 1}

A 43-year-old right handed male with past medical history of gun-shot wound, and nephrolithiasis presented to our medical center with symptoms of right hemiplegia and global aphasia (NIHSS 25) with unknown last known well (LKW). Vitals on admission were stable. Labs revealed Troponin of $49 \mathrm{ng} / \mathrm{ml}$, with ST elevation in leads II, III, aVF, V2-6 (Fig. 2). Left heart coronary (LHC) angiography revealed $60 \%$ stenosis with associated non-atherosclerotic

Table 1 Summary and comparison of patient presentation, management, and outcomes

\begin{tabular}{|c|c|c|c|}
\hline & Patient 1 & Patient 2 & Patient 3 \\
\hline Age & 43 & 80 & 72 \\
\hline Gender & Male & Female & Male \\
\hline Past medical history & Nephrolithiasis, gun shot wound & COPD, tobacco use & Pancreatitis \\
\hline CTH findings & Acute left MCA infarction & Acute Left MCA Infarction & Acute left MCA infarction \\
\hline CTA brain findings & Left M1 thrombus & Left M1 thrombus & Left M1 thrombus \\
\hline CT Perfusion findings & $\begin{array}{l}>\mathrm{CBF}<30 \% \text { volume: } 55 \mathrm{cc} \\
>\text { Tmax }>6.0 \text { s volume: } 96 \mathrm{cc} \\
>\text { Mismatch volume: } 41 \mathrm{cc} \\
>\text { Mismatch ratio: } 1.7\end{array}$ & $\begin{array}{l}>\mathrm{CBF}<30 \% \text { volume: } 28 \mathrm{cc} \\
>\mathrm{Tmax}>6.0 \mathrm{~s} \text { volume: } 36 \mathrm{cc} \\
>\text { Mismatch volume: } 8 \mathrm{cc} \\
>\text { Mismatch ratio: } 1.3\end{array}$ & $\begin{array}{l}>\mathrm{CBF}<30 \% \text { volume: } 12 \mathrm{cc} \\
>\mathrm{Tmax}>6.0 \mathrm{~s} \text { volume: } 48 \mathrm{cc} \\
\text { > Mismatch volume: } 36 \mathrm{cc} \\
>\text { Mismatch ratio: } 4\end{array}$ \\
\hline NIHSS on presentation & 25 & 23 & 17 \\
\hline EKG findings & $\begin{array}{l}\text { Anterior inferior STEMI in leads II, III, } \\
\text { aVF, V2-6 }\end{array}$ & Anterior STEMI in leads V3-V5 & Anteroseptal STEMI \\
\hline Troponin level & $49 \mathrm{ng} / \mathrm{ml}$ & $1.1 \mathrm{ng} / \mathrm{ml}$ & $6.5 \mathrm{ng} / \mathrm{ml}$ \\
\hline Echo and LHC findings & $\begin{array}{l}\text { Echo: EF } 30 \% \text { and LV thrombus } \\
\text { LHC: distal LAD non-artherosclerotic } \\
\text { thrombus }\end{array}$ & $\begin{array}{l}\text { Echo: EF } 29 \% \text { and LV thrombus } \\
\text { LHC: N/A }\end{array}$ & $\begin{array}{l}\text { Echo: EF } 30 \% \text { and LV thrombus } \\
\text { LHC: N/A }\end{array}$ \\
\hline \multicolumn{4}{|l|}{ Intervention } \\
\hline Medical & $\begin{array}{l}>\text { No intravenous alteplase } \\
>\text { Received non-bolus neuro-scale } \\
\text { heparin } \\
>\text { Started long-term aspirin } \\
>\text { Started long-term statin }\end{array}$ & $\begin{array}{l}>\text { Received intravenous alteplase } \\
>\text { No heparin } \\
>\text { Started long-term aspirin } \\
>\text { Started long-term statin }\end{array}$ & $\begin{array}{l}>\text { No intravenous alteplase } \\
>\text { Received non-bolus neuro } \\
\text { scale heparin drip } \\
>\text { Started long-term aspi- } \\
\text { rin + clopidogrel } \\
>\text { Started long-term statin }\end{array}$ \\
\hline Surgical & $\begin{array}{l}>\text { PCI without intervention/stenting } \\
>\text { No mechanical thrombectomy }\end{array}$ & $\begin{array}{l}>\text { No PCI } \\
>\text { No mechanical thrombectomy }\end{array}$ & $\begin{array}{l}>\text { No PCI } \\
>\text { No mechanical thrombectomy }\end{array}$ \\
\hline Outcome & $\begin{array}{l}\text { Transitioned to comfort care } \\
\text { died on hospital day } 7\end{array}$ & $\begin{array}{l}\text { Transitioned to comfort care and dis- } \\
\text { charged to hospice. Died } 3 \text { days after } \\
\text { discharge }\end{array}$ & $\begin{array}{l}\text { Transitioned to comfort care } \\
\text { Died on hospital day } 7\end{array}$ \\
\hline
\end{tabular}

$C T H$ computed tomography of head, CTA brain computed tomography angiogram of brain, EKG electrocardiogram, Echo echocardiogram, $L H C$ left heart catheterization, $L A D$ left anterior descending, $L V$ left ventricle, $P C I$ percutaneous intervention 

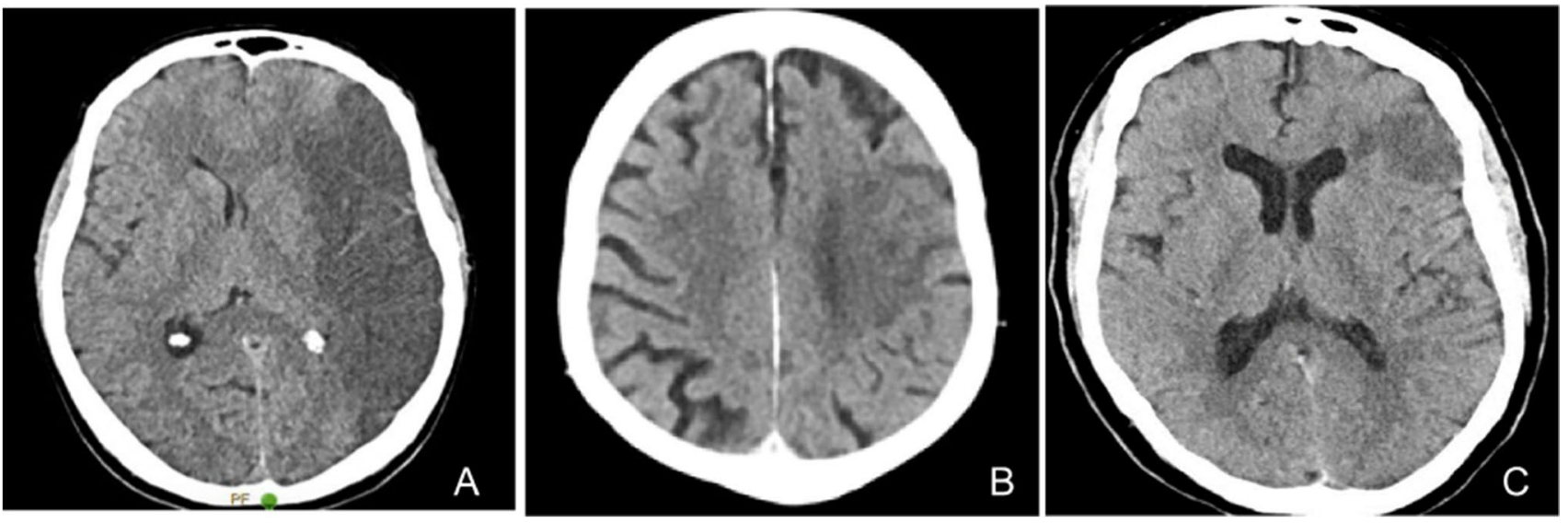

Fig. 1 CT Head imaging on admission showing evolving acute left MCA infarction in patient 1 (A), patient 2 (B), and patient 3 (C)

Fig. 2 EKG showing anteriorinferior STEMI in patient $1(\mathbf{A})$, anterior STEMI in patient 2 (B), and anterior STEMI in patient $3(\mathbf{C})$

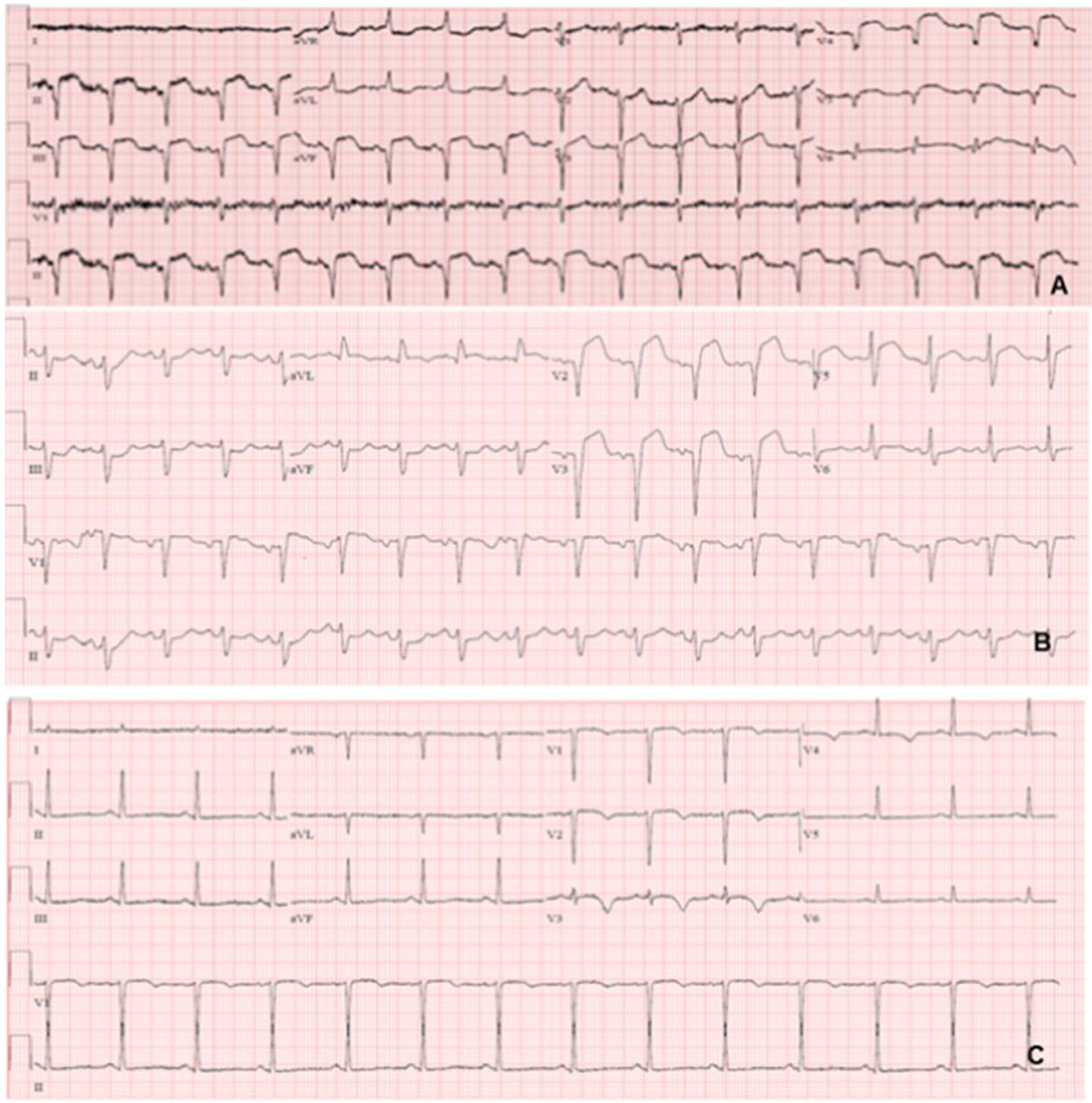

thrombus in the distal left anterior descending (LAD) artery. Echocardiogram revealed left ventricle (LV) apical thrombus with ejection fraction (EF) of $30 \%$. CT cerebral angiogram (CTA) showed a long segment intraluminal thrombus within the left M1 segment with distal M1 occlusion. CT perfusion showed core of $55 \mathrm{cc}$ with a mismatch volume and ratio of $41 \mathrm{cc}$ and 1.7 respectively.

Given unknown LKW, with unavailability of MRI FLAIR-diffusion mismatch protocol at our institution, acute management ischemic stroke did not include alteplase, nor 
MT. Due to STEMI on presentation, he was loaded with aspirin and Ticagrelor, with only aspirin continued after, and taken for emergent percutaneous coronary angioplasty. PCI was aborted due to need of bolus heparin prior to catheterization, and dual anti-platelet therapy "DAPT" post- catheterization, which would put him at increased risk of hemorrhagic conversion. Subsequent medical management was optimized for acute ischemia with goal directed medical therapy (GDMT) including aspirin. Immediate anticoagulation was not started for LV thrombus given risk of hemorrhagic conversion of large acute ischemic stroke. On day 5 of admission, "neuro scale" heparin drip (aPTT goal 52-75, no bolus) was started for LV thrombus, without transitioning to "cardiac scale" (aPTT goal of 72-95). His hospital course was further complicated by wide complex tachycardia secondary to known LAD occlusion, which was medically managed with lidocaine and amiodarone. He was ultimately transitioned to comfort care and passed away on day 7 of admission.

\section{Case presentation 2}

An 80-year-old right handed female with past medical history of COPD, and tobacco use presented initially to an outside hospital with right hemiplegia and global aphasia (NIHSS 23) with LKW $\leq 4.5 \mathrm{~h}$. She was given alteplase at $3.2 \mathrm{mg}$ bolus with infusion at $28.7 \mathrm{mg} / \mathrm{h}$, and transferred to OSU for further evaluation. On arrival, CTA revealed left M1 occlusion and CT Perfusion revealed core of $28 \mathrm{cc}$, mismatch volume and ratio of $8 \mathrm{cc}$ and 1.3 respectively. She was deemed to not be a MT candidate given her low mismatch ratio. EKG revealed an acute STEMI with mild elevation of Troponin to $1.1 \mathrm{ng} / \mathrm{ml}$ and ST elevation in leads V3-5 (Fig. 2). Echocardiography showed an EF of $29 \%$ and apical LV thrombus.

Stroke management included intravenous (IV) alteplase $(0.9 \mathrm{mg} / \mathrm{kg})$, aspirin at $24-\mathrm{h}$ post alteplase, statin, blood pressure control, and preventing edema by induced hypernatremia (140-145 mmol/L) using 0.9\% normal saline. Due to down-trending troponin, lack of new EKG changes, and the need for DAPT increasing the risk of hemorrhagic conversion, she was not offered PCI, nor started on a heparin drip. Her AMI was managed with GDMT. Given her comorbidities, age, and wishes, she was transitioned to comfort care and discharged to hospice with NIHSS of 25. She passed away at day 3 of discharge.

\section{Case presentation 3}

A 72-year-old right handed male with past medical history of pancreatitis presented with right hemiplegia and facial droop (NIHSS 27) with LKW $>4.5 \mathrm{~h}$. Vitals were pertinent for tachycardia to 110 on admission. Labs revealed Troponin of $6.5 \mathrm{ng} / \mathrm{ml}$. His EKG showed an acute STEMI in the anteroseptal region (Fig. 2). CTA showed a Left M1 occlusion. CT perfusion showed a core of $12 \mathrm{cc}$ with mismatch volume and ratio of $36 \mathrm{cc}$ and 4.0 respectively. Echocardiogram revealed an $\mathrm{EF} 30 \%$ and LV thrombus. As with the cases above, etiology of acute cerebral and myocardial ischemia was presumed secondary to his LV thrombus.

His AIS was managed medically with dual anti-platelet with aspirin/ clopidogrel, and statin. He did not receive alteplase due to being outside the window, nor did he get mechanical thrombectomy due to inability to perform the procedure per neurosugery. His AMI was managed medically, with non-bolus "neuro scale" heparin drip and antiplatelet and statin. His hospital course was complicated by ventricular tachycardia. He continued to have significant neurological disability with significant dysarthria and dysphagia requiring PEG tube. Family elected to transition him to comfort care and he passed away on hospital day 7 .

\section{Discussion}

\section{Epidemiology}

The incidence and prevalence of simultaneous AIS and AMI are unknown due to rarity of presentation. The reported incidences in literature are from observational studies and case reports. Rokey et al. found 58\% prevalence of CAD in patients presenting with AIS/ TIA [6]. Chin et al. reported the incidence of CCI as $12.7 \%$ in geriatric patients who were screened for AMI within $72 \mathrm{~h}$ of admission for acute stroke [7]. Findings from the Global Registry of Acute Coronary Event (GRACE) trial reported an incidence of in-hospital stroke as $0.9 \%$ in a cohort of patients presenting with acute coronary syndrome, with a higher incidence in STEMI compared to non-STEMI [8]. The most recent data in 2017 from Yeo et al. showed that $6 \%$ of patients with acute stroke had ST segment elevation $[9,10]$. Notably, these reported incidences are mostly for non-synchronous presentation, rather than simultaneous presentation of AIS and AMI.

\section{Pathophysiology and pathogenesis}

There are four major pathophysiologic mechanisms of CCI including (1) cardioembolic, (2) neurogenic stunned myocardium, (3) hypotensive, and (4) dissection.

Cardioembolism to the cerebral and myocardial arteries can lead to AIS and AMI and is typically secondary to either LV thrombus formation associated with heart failure, atrial fibrillation, or paradoxical embolization via patent foramen ovale in patients with $\mathrm{RV}$ thrombus or deep vein thrombosis $[9,11-13]$. 
Ischemic infarcts, especially ones involving the insular cortex, are associated with higher risk of neurogenic stunned myocardium and arrhythmias which can mimic ST-elevation myocardial ischemia $[14,15]$. This myocardial stunning is a result of dysfunction of CNS structures that control autonomic nervous system regulation of myocardium leading to sympathetic dominance. At the molecular level, there is reduced duration of delayed rectifier $\mathrm{K}^{+}$channels and alteration in L-type $\mathrm{Ca}^{++}$channels. Alterations of $\mathrm{K}^{+}$ channels lead to shortened diastolic interval (DI), reduced action-potential duration (APD), and insufficient myocardial refractory period prior to generation of new stimulus. Concurrently, abnormal sympathetic nervous system (SNS) stimulation of L-type $\mathrm{Ca}^{++}$channels triggers frequent afterdepolarization. Ultimately, this affects myocardium rhythm and integrity, predisposing to wall motion abnormalities and formation of cardiac thrombus $[15,16]$.

Another reported mechanism of CCI is hypotension, which occurs as a sequela of AMI causing dysregulation of cerebral blood flow leading to watershed infarcts [3, 9]. Although rare, extension of an ascending aortic dissection to the coronary ostia and a subsequent extension to the carotid, vertebral, or basilar arteries may also explain the simultaneous occurrence of a cerebral and a coronary infarction [17].

\section{Management}

Management of AIS prioritizes thrombolytic administration and revascularization with MT. Alteplase is administered if ischemic stroke is within $4.5 \mathrm{~h}$ of LKW (Table 2), and MT is considered if there is a large vessel occlusion within $24 \mathrm{~h}$. Although less commonly used in the USA, tenectaplase, which is more fibrin-specific and has a longer mechanism of action, may be considered well [18].

Management of AMI is similar, prioritizing timely restoration of blood flow to coronary vessels using PCI and/or fibrinolytic therapy [22, 23]. PCI is the preferred method of revascularization when it can be performed within $120 \mathrm{~min}$, and in patients presenting with AMI within $12 \mathrm{~h}$. Fibrinolysis via tenecteplase, alteplase, or streptokinase is indicated for symptom onset within $12 \mathrm{~h}$ followed by transfer for PCI, or if PCI cannot be completed within 120 min (Table 2) [19, 20]. Peri-procedural heparin and dual antiplatelet therapy are recommended following PCI.

Management of CCI is individualized (Table 3). Among the twenty-five cases reviewed within the literature review performed, 7 (28\%) utilized MT, $15(60 \%)$ underwent PCI, $9(36 \%)$ received fibrinolysis (alteplase), and 12 (48\%) had concurrent cardiac thrombus. $4(16 \%)$ received mono antiplatelet (MAP) only, 3 (12\%) DAP only, 4 (16\%) MAP plus AC, $3(12 \%)$ DAP plus AC, 10 (40\%) heparin therapy, and $8(32 \%)$ AC other than, and/ or with heparin. Outcomes were reported in $16(64 \%)$, with $4(16 \%)$ and $6(24 \%)$ having MRS $\leq 3$ at 1 and 3 months respectively. Approximately $20 \%$ expired during hospitalization. This highlights the inconsistency in practice and outcomes, thus the need for clear guidelines.

\section{Stroke and LV thrombus}

The management of AIS in the setting of LV thrombus is centered on the decision of anticoagulation timing, since much of the cardiac literature recommends prompt treatment of thrombus. Management of LV thrombus includes anticoagulation therapy for 3-6 months with serial echocardiogram to monitor resolution of thrombus. Anticoagulation can be discontinued after 6 months if there is resolution of LV thrombus, low risk of thrombus re-formation, and no significant wall motion abnormality. Timing on initiation of $\mathrm{AC}$ in setting of $\mathrm{CCI}$ has no clear guidelines with variations as noted in Table 3.

\section{Controversies}

Dilemmas associated with management of $\mathrm{CCI}$ include the following:

Table 2 Dose of fibrinolytic therapy for acute ischemic stroke and acute myocardial ischemia [19-21]

\begin{tabular}{lll}
\hline & Acute ischemic stroke & Acute myocardial ischemia \\
\hline Alteplase & $-0.9 \mathrm{mg} / \mathrm{kg}$ & $-15 \mathrm{mg}$ Bolus \\
& $-10 \%$ given as initial bolus over $1 \mathrm{~min}$ & - then $0.75 \mathrm{mg} / \mathrm{kg}(\mathrm{maximum} 50 \mathrm{mg})$ over $30 \mathrm{~min}$ \\
& $-90 \%$ as a continuous infusion over $60 \mathrm{~min}$ & - then $0.5 \mathrm{mg} / \mathrm{kg}(\mathrm{maximum} 35 \mathrm{mg}$ ) over the next $60 \mathrm{~min}$ \\
Tenecteplase & $-0.25-0.4 \mathrm{mg} / \mathrm{kg}$ & - Single bolus over 5 to $10 \mathrm{~s}$ based upon body weight: \\
& - consider $0.25 \mathrm{mg} / \mathrm{kg}$ for LVO & $-<60 \mathrm{~kg}: 30 \mathrm{mg}$ \\
& - consider $0.4 \mathrm{mg} / \mathrm{kg}$ for minor, non- LVO & -60 to $69 \mathrm{~kg}: 35 \mathrm{mg}$ \\
& & -70 to $79 \mathrm{~kg}: 40 \mathrm{mg}$ \\
Reteplase & - Not indicated & -80 to $89 \mathrm{~kg}: 45 \mathrm{mg}$ \\
\hline
\end{tabular}

LVO large vessel occlusion 
Table 3 Approach to cardio- cerebral infarct and outcomes from worldwide reported cases [9, 24-39]

\begin{tabular}{|c|c|c|c|c|c|c|c|c|c|}
\hline Case & Sex/age & $\begin{array}{l}\text { CTH/CTA } \\
\text { findings }\end{array}$ & NIHSS & Fibrinolysis & PCI & MT & AC/AP & $\begin{array}{l}\text { Cardiac } \\
\text { thrombus }\end{array}$ & Outcome \\
\hline $\begin{array}{c}\text { Eskandarani } \\
2021 \text { [26] }\end{array}$ & $\begin{array}{c}62 \text { years } \\
\text { old/M }\end{array}$ & $\begin{array}{l}\text { LCCA occlu- } \\
\text { sion }\end{array}$ & 22 & $\begin{array}{l}\text { None } \\
\text { reported }\end{array}$ & $\begin{array}{l}\text { None } \\
\text { reported }\end{array}$ & $\begin{array}{l}\text { None } \\
\text { reported }\end{array}$ & $\begin{array}{l}\text { ASA loading } \\
\text { dose } \\
\text { Clopidogrel } \\
\text { loading dose } \\
\text { Continued } \\
\text { with DAP }\end{array}$ & $\begin{array}{l}\text { None } \\
\text { reported }\end{array}$ & Death \\
\hline $\begin{array}{c}\text { Eskandarani } \\
2021 \text { [26] }\end{array}$ & $\begin{array}{c}50 \text { years } \\
\text { old/M }\end{array}$ & $\begin{array}{l}\text { BL cerebral } \\
\text { ischemia }\end{array}$ & 21 & $\begin{array}{l}\text { None } \\
\text { reported }\end{array}$ & $\begin{array}{l}\text { None } \\
\text { reported }\end{array}$ & $\begin{array}{l}\text { None } \\
\text { reported }\end{array}$ & $\begin{array}{l}\text { MAP } \\
\text { AC (unknown } \\
\text { type and } \\
\text { dosage) }\end{array}$ & $\begin{array}{l}\text { LV throm- } \\
\text { bus }\end{array}$ & $\begin{array}{l}\text { None } \\
\text { reported }\end{array}$ \\
\hline $\begin{array}{l}\text { Iqbal } 2021 \\
{[30]}\end{array}$ & $\begin{array}{c}65 \text { years } \\
\text { old/M }\end{array}$ & $\begin{array}{l}\text { LMCA } \\
\text { ischemia }\end{array}$ & $\begin{array}{l}\text { None } \\
\text { reported }\end{array}$ & $\begin{array}{l}\text { IV alteplase } \\
\text { at } 0.9 \mathrm{mg} / \\
\mathrm{kg} \text { (discon- } \\
\text { tinued after } \\
\text { bolus due } \\
\text { to bleed- } \\
\text { ing) }\end{array}$ & $\begin{array}{l}\text { None } \\
\text { reported }\end{array}$ & $\begin{array}{l}\text { None } \\
\text { reported }\end{array}$ & $\begin{array}{l}\text { DAP } \\
\text { LMWH }\end{array}$ & $\begin{array}{l}\text { LV throm- } \\
\text { bus }\end{array}$ & $\begin{array}{l}\text { None } \\
\text { reported }\end{array}$ \\
\hline Ibekwe 2021 & $\begin{array}{c}43 \text { years } \\
\text { old/M }\end{array}$ & $\begin{array}{l}\text { LM1 occlu- } \\
\text { sion }\end{array}$ & 25 & $\begin{array}{l}\text { None } \\
\text { reported }\end{array}$ & $\begin{array}{l}\text { None } \\
\text { reported }\end{array}$ & $\begin{array}{l}\text { None } \\
\text { reported }\end{array}$ & $\begin{array}{l}\text { ASA } 325 \mathrm{mg} \\
\text { load } \\
\text { Ticagrelor } \\
\text { load } \\
\text { MAP after } \\
\text { Heparin } \\
\text { infusion on } \\
\text { day } 5\end{array}$ & $\begin{array}{l}\text { LV throm- } \\
\text { bus }\end{array}$ & Death \\
\hline Ibekwe 2021 & $\begin{array}{c}80 \text { years } \\
\text { old } / F\end{array}$ & $\begin{array}{l}\text { LM1 occlu- } \\
\text { sion }\end{array}$ & 23 & $\begin{array}{l}\text { IV alteplase } \\
\text { at } 039 \mathrm{mg} / \\
\mathrm{kg}\end{array}$ & $\begin{array}{l}\text { None } \\
\text { reported }\end{array}$ & $\begin{array}{l}\text { None } \\
\text { reported }\end{array}$ & ASA & $\begin{array}{l}\text { LV throm- } \\
\text { bus }\end{array}$ & Death \\
\hline Ibekwe 2021 & $\begin{array}{c}72 \text { years } \\
\text { old/M }\end{array}$ & $\begin{array}{l}\text { LM1 occlu- } \\
\text { sion }\end{array}$ & 27 & $\begin{array}{l}\text { None } \\
\text { reported }\end{array}$ & $\begin{array}{l}\text { None } \\
\text { reported }\end{array}$ & $\begin{array}{l}\text { None } \\
\text { reported }\end{array}$ & $\begin{array}{l}\text { DAP } \\
\text { Heparin infu- } \\
\text { sion }\end{array}$ & $\begin{array}{l}\text { LV throm- } \\
\text { bus }\end{array}$ & Death \\
\hline Abe 2019 & $\begin{array}{c}73 \text { years } \\
\text { old } / F\end{array}$ & & 21 & $\begin{array}{l}\text { IV alteplase } \\
\text { at } 0.6 \mathrm{mg} / \\
\mathrm{kg}\end{array}$ & $\begin{array}{l}\text { Yes, no } \\
\text { stenting }\end{array}$ & Yes & $\begin{array}{l}\text { Rivaroxaban } \\
\text { on day } 5\end{array}$ & $\begin{array}{l}\text { None } \\
\text { reported }\end{array}$ & $\begin{array}{r}\text { MRS } 2 \text { at } \\
3 \text { month }\end{array}$ \\
\hline $\begin{array}{l}\text { Katsuki } \\
2019 \text { [37] }\end{array}$ & $\begin{array}{c}72 \text { years } \\
\text { old/M }\end{array}$ & $\begin{array}{l}\text { Lt Cerebellar } \\
\text { ischemia }\end{array}$ & 29 & $\begin{array}{l}\text { None } \\
\text { reported }\end{array}$ & $\begin{array}{l}\text { Yes, without } \\
\text { stenting }\end{array}$ & $\begin{array}{l}\text { None } \\
\text { reported }\end{array}$ & $\begin{array}{l}\text { Heparin infu- } \\
\text { sion }\end{array}$ & $\begin{array}{l}\text { LAA throm- } \\
\text { bus }\end{array}$ & $\begin{array}{l}\text { None } \\
\text { reported }\end{array}$ \\
\hline $\begin{array}{l}\text { Sakuta } 2019 \\
\text { [36] }\end{array}$ & $\begin{array}{l}55 \text { years } \\
\text { old/F }\end{array}$ & $\begin{array}{l}\text { LMCA } \\
\text { occlusion }\end{array}$ & 23 & $\begin{array}{l}\text { None } \\
\text { reported }\end{array}$ & $\begin{array}{l}\text { Yes, without } \\
\text { stenting }\end{array}$ & Yes & $\begin{array}{l}\text { Heparin infu- } \\
\text { sion }\end{array}$ & $\begin{array}{l}\text { None } \\
\text { reported }\end{array}$ & $\begin{array}{l}\text { MRS } 3 \text { at } \\
3 \text { month }\end{array}$ \\
\hline $\begin{array}{c}\text { Wan Asyraf } \\
2019 \text { [39] }\end{array}$ & $\begin{array}{c}33 \text { years } \\
\text { old/M }\end{array}$ & $\begin{array}{l}\text { LMCA } \\
\text { occlusion }\end{array}$ & 11 & $\begin{array}{l}\text { IV Tenect- } \\
\text { eplase }\end{array}$ & $\begin{array}{l}\text { None } \\
\text { reported }\end{array}$ & $\begin{array}{l}\text { None } \\
\text { reported }\end{array}$ & $\begin{array}{l}\text { Heparin infu- } \\
\text { sion } \\
\text { DAP }\end{array}$ & $\begin{array}{l}\text { None } \\
\text { reported }\end{array}$ & $\begin{array}{l}\text { MRS } 1 \text { at } \\
1 \text { month }\end{array}$ \\
\hline $\begin{array}{l}\text { Plata } 2018 \\
\text { [27] }\end{array}$ & $\begin{array}{c}46 \text { years } \\
\text { old/M }\end{array}$ & $\begin{array}{l}\text { RM2 occlu- } \\
\text { sion }\end{array}$ & 15 & $\begin{array}{l}\text { IA alteplase } \\
\text { at } 5 \mathrm{mg} \\
\text { Bolus }\end{array}$ & $\begin{array}{l}\text { Yes with } \\
\text { Drug Elut- } \\
\text { ing Stent }\end{array}$ & Yes & $\begin{array}{l}\text { MAP } \\
\text { Warfarin }\end{array}$ & $\begin{array}{l}\mathrm{LV} \\
\text { thrombus }\end{array}$ & $\begin{array}{l}\text { MRS } 0 \text { at } \\
1 \text { month }\end{array}$ \\
\hline Yeo 2017 [9] & $\begin{array}{c}45 \text { years } \\
\text { old } / \mathrm{M}\end{array}$ & $\begin{array}{l}\text { RICA occlu- } \\
\text { sion }\end{array}$ & $\begin{array}{l}\text { None } \\
\text { reported }\end{array}$ & $\begin{array}{l}\text { None } \\
\text { reported }\end{array}$ & $\begin{array}{l}\text { Yes with } \\
\text { Drug Elut- } \\
\text { ing Stent }\end{array}$ & Yes & $\begin{array}{l}\text { Heparin } \\
\text { bridge to } \\
\text { Warfarin }\end{array}$ & $\begin{array}{l}\mathrm{LV} \\
\text { throm } \\
\text { bus }\end{array}$ & $\begin{array}{l}\text { MRS } 3 \text { at } \\
3 \text { month }\end{array}$ \\
\hline Yeo 2017 [9] & $\begin{array}{c}53 \text { years } \\
\text { old/M }\end{array}$ & $\begin{array}{l}\text { LMCA } \\
\text { occlusion }\end{array}$ & $\begin{array}{l}\text { None } \\
\text { reported }\end{array}$ & $\begin{array}{l}\text { None } \\
\text { reported }\end{array}$ & $\begin{array}{l}\text { Yes with } \\
\text { Bare- } \\
\text { Metal } \\
\text { Stent }\end{array}$ & Yes & ASA $81 \mathrm{mg}$ & $\begin{array}{l}\text { None } \\
\text { reported }\end{array}$ & $\begin{array}{l}\text { MRS } 4 \\
\text { at } 3 \\
\text { month }\end{array}$ \\
\hline Yeo 2017 [9] & $\begin{array}{c}71 \text { years } \\
\text { old/F }\end{array}$ & $\begin{array}{l}\text { RPCA occlu- } \\
\text { sion }\end{array}$ & $\begin{array}{l}\text { None } \\
\text { reported }\end{array}$ & $\begin{array}{l}\text { None } \\
\text { reported }\end{array}$ & Yes & Yes & None reported & $\begin{array}{l}\mathrm{LV} \\
\text { thrombus }\end{array}$ & $\begin{array}{l}\text { None } \\
\text { reported }\end{array}$ \\
\hline
\end{tabular}


Table 3 (continued)

\begin{tabular}{|c|c|c|c|c|c|c|c|c|c|}
\hline Case & Sex/age & $\begin{array}{l}\text { CTH/CTA } \\
\text { findings }\end{array}$ & NIHSS & Fibrinolysis & PCI & MT & $\mathrm{AC} / \mathrm{AP}$ & $\begin{array}{l}\text { Cardiac } \\
\text { thrombus }\end{array}$ & Outcome \\
\hline $\begin{array}{l}\text { Yeo } 2017 \\
\text { [9] }\end{array}$ & $\begin{array}{l}\text { Middle } \\
\text { age/M }\end{array}$ & $\begin{array}{l}\text { LMCA } \\
\text { occlusion }\end{array}$ & $\begin{array}{l}\text { None } \\
\text { reported }\end{array}$ & $\begin{array}{l}\text { IV alteplase } \\
\text { at } 0.9 \mathrm{mg} / \\
\mathrm{kg}\end{array}$ & $\begin{array}{l}\text { None } \\
\text { reported }\end{array}$ & $\begin{array}{l}\text { None } \\
\text { reported }\end{array}$ & None reported & $\begin{array}{l}\text { None } \\
\text { reported }\end{array}$ & $\begin{array}{l}\text { MRS 1 } \\
\text { at } 3 \\
\text { month }\end{array}$ \\
\hline $\begin{array}{l}\text { Kijpaisalra- } \\
\text { tana } 2017 \\
{[25]}\end{array}$ & $\begin{array}{c}65 \text { years } \\
\text { old/M }\end{array}$ & $\begin{array}{l}\text { RM1 occlu- } \\
\text { sion }\end{array}$ & 12 & $\begin{array}{l}\text { IV alteplase } \\
\text { at } 0.9 \mathrm{mg} / \\
\mathrm{kg}\end{array}$ & $\begin{array}{l}\text { Yes with } \\
\text { DES }\end{array}$ & $\begin{array}{l}\text { None } \\
\text { reported }\end{array}$ & $\begin{array}{l}\text { DAP with } \\
\text { ASA/ Clopi- } \\
\text { dogrel }\end{array}$ & $\begin{array}{l}\text { None } \\
\text { reported }\end{array}$ & $\begin{array}{l}\text { None } \\
\text { reported }\end{array}$ \\
\hline $\begin{array}{l}\text { Hosoya } \\
2017 \text { [28] }\end{array}$ & $\begin{array}{c}50 \text { years } \\
\text { old/M }\end{array}$ & $\begin{array}{l}\text { Multifocal } \\
\text { Ischemia }\end{array}$ & $\begin{array}{l}\text { None } \\
\text { reported }\end{array}$ & $\begin{array}{l}\text { None } \\
\text { reported }\end{array}$ & $\begin{array}{l}\text { Yes, no } \\
\text { stenting }\end{array}$ & $\begin{array}{l}\text { None } \\
\text { reported }\end{array}$ & ASA & $\begin{array}{l}\text { None } \\
\text { reported }\end{array}$ & $\begin{array}{l}\text { None } \\
\text { reported }\end{array}$ \\
\hline $\begin{array}{l}\text { Tokuda } \\
2016[35]\end{array}$ & $\begin{array}{c}87 \text { years } \\
\text { old/F }\end{array}$ & $\begin{array}{l}\text { RMCA } \\
\text { and ACA } \\
\text { occlusion }\end{array}$ & 19 & $\begin{array}{l}\text { None } \\
\text { reported }\end{array}$ & Yes & Yes & Rivaroxaban & $\begin{array}{l}\text { None } \\
\text { reported }\end{array}$ & $\begin{array}{l}\text { MRS } 3 \\
\text { at } 3 \\
\text { month }\end{array}$ \\
\hline $\begin{array}{l}\text { Maciel } 2015 \\
{[24]}\end{array}$ & $\begin{array}{c}44 \text { years } \\
\text { old/M }\end{array}$ & $\begin{array}{l}\text { RMCA } \\
\text { ischemia }\end{array}$ & 11 & $\begin{array}{l}\text { IV alteplase } \\
\text { at } 0.9 \mathrm{mg} / \\
\mathrm{kg}\end{array}$ & $\begin{array}{l}\text { None } \\
\text { reported }\end{array}$ & $\begin{array}{l}\text { None } \\
\text { reported }\end{array}$ & None reported & $\begin{array}{l}\text { None } \\
\text { reported }\end{array}$ & $\begin{array}{l}\text { MRS } 2 \text { at } \\
3 \text { months }\end{array}$ \\
\hline $\begin{array}{l}\text { Wee } 2015 \\
\text { [29] }\end{array}$ & $\begin{array}{c}49 \text { years } \\
\text { old/M }\end{array}$ & $\begin{array}{l}\text { RPCA } \\
\text { ischemia }\end{array}$ & $\begin{array}{l}\text { None } \\
\text { reported }\end{array}$ & $\begin{array}{l}\text { None } \\
\text { reported }\end{array}$ & $\begin{array}{l}\text { Yes, no } \\
\text { stenting }\end{array}$ & $\begin{array}{l}\text { None } \\
\text { reported }\end{array}$ & $\begin{array}{l}\text { Clopidogrel } \\
300 \text { mg load } \\
\text { Heparin infu- } \\
\text { sion at } 48 \mathrm{~h} \\
\text { (apt goal 60) } \\
\text { Warfarin on } \\
\text { Day } 3\end{array}$ & $\begin{array}{l}\text { LV throm- } \\
\text { bus }\end{array}$ & $\begin{array}{c}\text { MRS } 2 \\
\text { at } 1 \\
\text { month }\end{array}$ \\
\hline $\begin{array}{l}\text { Gonzalez- } \\
\text { Pacheco } \\
2014 \text { [33] }\end{array}$ & $\begin{array}{l}66 \text { years } \\
\text { old/F }\end{array}$ & $\begin{array}{l}\text { Rt FrontoPa- } \\
\quad \text { rietal } \\
\text { Ischemia }\end{array}$ & 16 & $\begin{array}{l}\text { IV alteplase } \\
\text { at } 0.9 \mathrm{mg} / \\
\mathrm{kg}\end{array}$ & $\begin{array}{l}\text { Yes with } \\
\text { DES }\end{array}$ & $\begin{array}{l}\text { None } \\
\text { reported }\end{array}$ & $\begin{array}{l}\text { ASA } 81 \mathrm{mg} \text { at } \\
48 \mathrm{~h} \\
\text { DAP with } \\
\text { clopidogrel } \\
\text { / ASA on } \\
\text { day } 4\end{array}$ & $\begin{array}{l}\text { LV throm- } \\
\text { bus }\end{array}$ & $\begin{array}{l}\text { None } \\
\text { reported }\end{array}$ \\
\hline $\begin{array}{l}\text { Hashimoto } \\
2014 \text { [34] }\end{array}$ & $\begin{array}{c}84 \text { years } \\
\text { old/M }\end{array}$ & $\begin{array}{r}\text { Multifocal } \\
\text { ischemia }\end{array}$ & 1 & $\begin{array}{l}\text { None } \\
\text { reported }\end{array}$ & $\begin{array}{l}\text { Yes, without } \\
\text { stenting }\end{array}$ & $\begin{array}{l}\text { None } \\
\text { reported }\end{array}$ & $\begin{array}{l}\text { Heparin infu- } \\
\text { sion } \\
\text { Warfarin }\end{array}$ & $\begin{array}{l}\text { None } \\
\text { reported }\end{array}$ & MRS 0 \\
\hline $\begin{array}{l}\text { Kim } 2013 \\
{[32]}\end{array}$ & $\begin{array}{c}58 \text { years } \\
\text { old/M }\end{array}$ & $\begin{array}{l}\text { LMCA } \\
\text { ischemia }\end{array}$ & $\begin{array}{l}\text { None } \\
\text { reported }\end{array}$ & $\begin{array}{l}\text { None } \\
\text { reported }\end{array}$ & $\begin{array}{l}\text { Yes, with } \\
\text { DES }\end{array}$ & $\begin{array}{l}\text { None } \\
\text { reported }\end{array}$ & $\begin{array}{l}\text { Clopidogrel } \\
600 \mathrm{mg} \text { load }\end{array}$ & $\begin{array}{l}\text { None } \\
\text { reported }\end{array}$ & $\begin{array}{l}\text { None } r \\
\text { eported }\end{array}$ \\
\hline $\begin{array}{l}\text { Klecynski } \\
2012[31]\end{array}$ & $\begin{array}{c}62 \text { years } \\
\text { old/M }\end{array}$ & $\begin{array}{l}\text { BL Frontal } \\
\text { and Lt } \\
\text { Parietal } \\
\text { ischemia }\end{array}$ & $\begin{array}{l}\text { None } \\
\text { reported }\end{array}$ & $\begin{array}{l}\text { None } \\
\text { reported }\end{array}$ & $\begin{array}{l}\text { Yes, no } \\
\text { stenting }\end{array}$ & $\begin{array}{l}\text { None } \\
\text { reported }\end{array}$ & $\begin{array}{l}\text { Clopidogrel } \\
600 \mathrm{mg} \text { load } \\
\text { ASA } 300 \mathrm{mg} \\
\text { load } \\
\text { Heparin bolus } \\
\text { and infusion } \\
\text { Warfarin }\end{array}$ & $\begin{array}{l}\text { LAA throm- } \\
\text { bus }\end{array}$ & $\begin{array}{l}\text { None } \\
\text { reported }\end{array}$ \\
\hline $\begin{array}{l}\text { Omar } 2010 \\
{[3]}\end{array}$ & $\begin{array}{c}48 \text { years } \\
\text { old/M }\end{array}$ & $\begin{array}{l}\text { BL occipital } \\
\text { and } \\
\text { cerebellar } \\
\text { ischemia }\end{array}$ & 25 & $\begin{array}{l}\text { None } \\
\text { reported }\end{array}$ & $\begin{array}{l}\text { None } \\
\text { reported }\end{array}$ & $\begin{array}{l}\text { None } \\
\text { reported }\end{array}$ & None reported & $\begin{array}{l}\text { None } \\
\text { reported }\end{array}$ & Death \\
\hline
\end{tabular}

$A C$ anti-coagulation, $A P$ anti-platelet, ASA aspirin, $C T H$ computed tomography of head, $C T A$ computed tomography angiogram, $D A P$ dual antiplatelet, $D E S$ drug eluting stent, $I A$ intra-arterial, $I C H$ intra cerebral hemorrhage, $I V$ intra-venous, $L A A$ left atrial appendage, $L M W H$ low molecular weight heparin, $L V$ left ventricle, NIHSS National Institute of Health Stroke Scale, MAP mono anti-platelet, MRS Modified Rankin Score, $M T$ mechanical thrombectomy, $P C I$ per-cutaneous intervention, $P E A$ pulseless electrical activity 
1. The different dosages and duration of IV alteplase for management of AIS versus AMI (Table 2).

2. The use of PCI with stenting requires peri-procedural heparin infusion, and prolonged use of dual-anti platelet therapy. This has been associated with increased risk of hemorrhagic conversion in AIS, but evidence is limited regarding long-term clinical outcomes [40].

3. Caution with use of thrombolytics in the setting of acute STEMI due to the risk of myocardial rupture [41].

\section{Risk of hemorrhagic transformation}

Currently, data on risk factors associated with hemorrhagic transformation in the setting of CCI is lacking. A small retrospective study from Schmidbauer et al. did not show increased incidence of ICH in AIS patients treated with PCI compared to those not getting PCI [42]. Several trials, such as Troponin Elevation in Acute Ischemic Stroke (TRELAS), which evaluated the diagnostic yield of troponin levels in AIS, did not report rates of symptomatic ICH. We await the results of ongoing trial Prediction of Acute Coronary Syndrome in Acute Ischemic Stroke (PRAISE), which is assessing the diagnostic yield of biomarkers, clinical scores, signs and symptoms, EKG, and echocardiography findings to identify patients in need of PCI. We hope that rates of hemorrhagic transformation are reported.

Given the lack of high quality data, clinicians should individualize management-prioritizing fibrinolytic therapy and mechanical revascularization-with full assessment of bleeding risk, and other comorbidities.

\section{Treatment strategies}

Regarding CCI necessitating acute intervention, we propose combined treatment of myocardial and cerebral vascular territories with administration of alteplase $(0.9 \mathrm{mg} /$ $\mathrm{kg}$ ) followed by PCI with stenting if indicated [9]. Concomitant need for mechanical cerebral thrombectomy can then be assessed using cerebral angiogram. Another approach could be combining mechanical thrombectomy with PCI in select cases, with close monitoring of neurological status to detect symptomatic hemorrhagic conversion.

\section{Limitations}

This case series only reports observational findings and is not structured to evaluate for associations between different management options and clinical outcome (i.e., symptomatic ICH, morbidity/mortality). Furthermore, since all of the patients were transitioned to comfort care within a week of admission, it is impossible to predict overall outcomes. Lastly, the search strategy and inclusion criteria for the literature review focused on the current definition of CCI necessitating AMI and AIS within $48 \mathrm{~h}$ of each other, leading to exclusion of many studies.

\section{Conclusion}

Appropriate management of CCI poses a great challenge for practitioners. Clinicians must be mindful of the following: (1) treatment strategies to enable swift mechanical reperfusion to both the brain and myocardium; (2) risk of cardiac tamponade and myocardial rupture with intravenous thrombolytic therapy; (3) difference in dosage of thrombolytics for AIS versus AMI; and (4) risk of symptomatic intracerebral hemorrhage following administration of anticoagulation and/or antiplatelet therapy. There is an urgent need for future studies to guide optimal management.

Author contribution Dr. Hera Kamdar and Dr. Tamara Strohm were involved in manuscript review and editing. Dr. Elo Ibekwe is the main author of the manuscript.

\section{Declarations}

Ethics approval No ethics approval was necessary for this manuscript.

Informed consent Informed consent was obtained from health-care power of attorney over telephone.

Conflict of interest The authors declare no competing interests.

\section{References}

1. White H, Boden-Albala B, Wang C et al (2005) Ischemic stroke subtype incidence among whites, blacks, and Hispanics: the Northern Manhattan Study. Circulation 111:1327

2. Powers WJ, Rabinstein AA, Ackerson T et al (2019) Guidelines for the Early Management of Patients With Acute Ischemic Stroke: 2019 Update to the 2018 Guidelines for the Early Management of Acute Ischemic Stroke: A Guideline for Healthcare Professionals From the American Heart Association/American Stroke Association. Stroke 50:e344

3. Omar HR, Fathy A, Rashad R et al (2010) Concomitant acute right ventricular infarction and ischemic cerebrovascular stroke; possible explanations. Int Arch Med 3:25. https://doi.org/10.1186/ 1755-7682-3-25

4. Elsaid N, Mustafa W, Saied A (2020) Radiological predictors of hemorrhagic transformation after acute ischemic stroke: An evidence-based analysis. Neuroradiol J 33(2):118-133. https://doi. org/10.1177/1971400919900275 
5. Taku I, Li L et al (2019) Recent myocardial infarction is associated with increased risk in older adults with acute ischemic stroke receiving thrombolytic therapy. J Am Heart Assoc 8:e012450

6. Rokey R, Rolak LA, Harati Y et al (1984) Coronary artery disease in patients with cerebrovascular disease: a prospective study. Ann Neurol 16:50-53. https://doi.org/10.1002/ana.410160110

7. Chin PL, Kaminski J, Rout M (1977) Myocardial infarction coincident with cerebrovascular accidents in the elderly. Age Ageing 6:29-37. https://doi.org/10.1093/ageing/6.1.29

8. Budaj A, Flasinska K, Gore JM et al (2005) Magnitude of and risk factors for in-hospital and postdischarge stroke in patients with acute coronary syndromes: findings from a global registry of acute coronary events. Circulation 111:3242-3247. https://doi. org/10.1161/CIRCULATIONAHA.104.512806

9. Yeo LL, Andersson T, Yee KW et al (2017) Synchronous cardiocerebral infarction in the era of endovascular therapy: which to treat first? J Thromb Thrombolysis 44:104-111. https://doi.org/ 10.1007/s11239-017-1484-2

10. Fahad A, Sami A, Abdul T et al (2017) Incidence and outcomes of myocardial infarction in patients admitted with acute ischemic stroke. Stroke 48:2931-2938

11. Delewi R, Zijlstra F, Piek JJ (2012) Left ventricular thrombus formation after acute myocardial infarction. Heart 98:1743-1749. https://doi.org/10.1136/heartjnl-2012-301962

12. Kleber FX, Hauschild T, Schulz A et al (2017) Epidemiology of myocardial infarction caused by presumed paradoxical embolism via a patent foramen ovale. Circ J 81:1484-1489. https://doi.org/ 10.1253/circj.CJ-16-0995

13. Chlapoutakis GN, Kafkas NV, Katsanos SM et al (2010) Acute myocardial infarction and transient ischemic attack in a patient with lone atrial fibrillation and normal coronary arteries. Int $\mathbf{J}$ Cardiol 139:e1-e4. https://doi.org/10.1016/j.ijcard.2008.06.085

14. Laowattana S, Zeger SL, Lima JA et al (2006) Left insular stroke is associated with adverse cardiac outcome. Neurology 66:47783. https://doi.org/10.1212/01.wnl.0000202684.29640.60 (discussion 463)

15. Bersano A, Melchiorre P, Moschwitis G et al (2014) Tako-tsubo syndrome as a consequence and cause of stroke. Funct Neurol 29:135-137

16. Ibrahim MS, Samuel B, Mohamed W, Suchdev K (2019) Cardiac dysfunction in neurocritical care: an autonomic perspective. Neurocrit Care 30(3):508-521. https://doi.org/10.1007/ s12028-018-0636-3

17. Kawano H, Tomichi Y, Fukae S et al (2006) Aortic dissection associated with acute myocardial infarction and stroke found at autopsy. Intern Med 45:957-962. https://doi.org/10.2169/inter nalmedicine. 45.1589

18. Burgos A, Saver J (2019) Evidence that tenecteplase is non-inferior to alteplase for acute ischemic stroke. Stroke 50:2156-2162

19. Assessment of the Safety and Efficacy of a New Thrombolytic (ASSENT-2) Investigators, Van De Werf F, Adgey J et al (1999) Single-bolus tenecteplase compared with front-loaded alteplase in acute myocardial infarction: the ASSENT-2 double-blind randomised trial. Lancet 354(9180):716-722. https://doi.org/10. 1016/s0140-6736(99)07403-6

20. Topol EJ, Ohman EM, Armstrong PW, Wilcox R, Skene AM, Aylward P, Simes J, Dalby A, Betriu A, Bode C, White HD, Hochman JS, Emanuelson H, Vahanian A, Sapp S, Stebbins A, Moliterno DJ, Califf RM (2000) Survival outcomes 1 year after reperfusion therapy with either alteplase or reteplase for acute myocardial infarction: results from the Global Utilization of Streptokinase and t-PA for Occluded Coronary Arteries (GUSTO) III Trial. Circulation 102(15):1761-1765. https://doi.org/10.1161/01.cir.102.15. 1761

21. Warach S, Dula A, Milling T Jr (2020) Tenecteplase thrombolysis for acute ischemic stroke. Stroke 51:3440-3451
22. Ibanez B et al (2018) 2017 ESC Guidelines for the Management of acute myocardial infarction in patients presenting with STsegment elevation: the Task Force for the management of acute myocardial infarction in patients presenting with ST- segment elevation of the European Society of Cardiology (ESC). Eur Heart J 39(2):131-142

23. O'Gara PT, Kushner FG, Ascheim DD et al (2013) 2013 ACCF/ AHA guideline for the management of ST-elevation myocardial infarction: a report of the American College of Cardiology Foundation/American Heart Association Task Force on Practice Guidelines. Circulation 127:e362

24. Maciel R, Palma R, Sousa P et al (2015) Acute stroke with concomitant acute myocardial infarction: Will you thrombolyse? J Stroke 17:84-86. https://doi.org/10.5853/jos.2015.17.1.84

25. Kijpaisalratana N, Chutinet A, Suwanwela NC (2017) Hyperacute simultaneous cardiocerebral infarction: rescuing the brain or the heart first? Front Neurol 8:664. https://doi.org/10.3389/fneur. 2017.00664

26. Eskandarani R, Sahli S, Sawan S, Alsaeed A (2021) Simultaneous cardio-cerebral infarction in the coronavirus disease pandemic era: a case series. Medicine (Baltimore) 100(4):e24496. https:// doi.org/10.1097/MD.0000000000024496

27. Plata-Corona JC, Cerón-Morales JA, Lara-Solís B (2019) Nonhyperacute synchronous cardio-cerebral infarction treated by double intervensionist therapy. Cardiovasc Metab Sci 30(2):66-75

28. Hosoya H, Levine JJ, Henry DH, Goldberg S (2017) Double the trouble: acute coronary syndrome and ischemic stroke in polycythemia vera. Am J Med 130(6):e237-e240. https://doi.org/10. 1016/j.amjmed.2017.02.016

29. Wee CK, DivakarGosavi T, Huang W (2015) The clot strikes thrice: case report of a patient with 3 concurrent embolic events. Acta Neurol Taiwan 24(3):92-6

30. Iqbal $P$ (2021) The role of anticoagulation in post-COVID-19 concomitant stroke, myocardial infarction, and left ventricular thrombus: a case report. Am J Case Rep 22(1):1-6

31. Kleczyński P, Dziewierz A, Rakowski T, Rzeszutko L, Sorysz D, Legutko J, Dudek D (2012) Cardioembolic acute myocardial infarction and stroke in a patient with persistent atrial fibrillation. Int J Cardiol 161(3):e46-e47. https://doi.org/10.1016/j.ijcard. 2012.04.018

32. Kim HL, Seo JB, Chung WY, Zo JH, Kim MA, Kim SH (2013) Simultaneously presented acute ischemic stroke and non-st elevation myocardial infarction in a patient with paroxysmal atrial fibrillation. Korean Circ J 43(11):766-769. https://doi.org/10. 4070/kcj.2013.43.11.766

33. González-Pacheco H, Méndez-Domínguez A, Vieyra-Herrera G, Azar-Manzur F, Meave-González A, Rodríguez-Zanella H, Martínez-Sánchez C (2014) Reperfusion strategy for simultaneous ST-segment elevation myocardial infarction and acute ischemic stroke within a time window. Am J Emerg Med 32(9):1157.e1-4. https://doi.org/10.1016/j.ajem.2014.02.047

34. Hashimoto O, Sato K, Numasawa Y, Hosokawa J, Endo M (2014) Simultaneous onset of myocardial infarction and ischemic stroke in a patient with atrial fibrillation: multiple territory injury revealed on angiography and magnetic resonance. Int J Cardiol 172(2):e338-e340. https://doi.org/10.1016/j.ijcard.2013.12.312

35. Tokuda K, Shindo S, Yamada K, Shirakawa M, Uchida K, Horimatsu T, Ishihara M, Yoshimura S (2016) Acute embolic cerebral infarction and coronary artery embolism in a patient with atrial fibrillation caused by similar thrombi. J Stroke Cerebrovasc Dis 25(7):1797-1799. https://doi.org/10.1016/j.jstrokecerebrovasdis. 2016.01.055

36. Sakuta K, Mukai T, Fujii A, Makita K, Yaguchi H (2019) Endovascular therapy for concurrent cardio-cerebral infarction in a patient with trousseau syndrome. Front Neurol 10:965. https:// doi.org/10.3389/fneur.2019.00965 
37. Katsuki M, Katsuki S (2019) A case of cardiac tamponade during the treatment of simultaneous cardio-cerebral infarction associated with atrial fibrillation - case report. Surg Neurol Int. 10:241. https://doi.org/10.25259/SNI_504_2019

38. Abe S, Tanaka K, Yamagami H, Sonoda K, Hayashi H, Yoneda S, Toyoda K, Koga M (2019) Simultaneous cardio-cerebral embolization associated with atrial fibrillation: a case report. BMC Neurol 19(1):152. https://doi.org/10.1186/s12883-019-1388-1

39. Wan Asyraf WZ, Elengoe S, Che Hassan HH, Abu Bakar A, Remli R (2020) Concurrent stroke and ST-elevation myocardial infarction: is it a contraindication for intravenous tenecteplase? Med J Malaysia 75(2):169-170

40. Cucchiara B, Kasner SE, Tanne D et al (2009) Factors associated with intracerebral hemorrhage after thrombolytic therapy for ischemic stroke: pooled analysis of placebo data from the strokeacute ischemic NXY treatment (SAINT) I and SAINT II trials. Stroke 40:3067-3072. https://doi.org/10.1161/STROKEAHA.109.554386

41. Marto J, Kaupilla L, Jorge C et al (2019) Intravenous thromblysis for acute ischemic stroke after recent myocardial infarction. Case Series and Systematic Review. Stroke 50:2813-2818. https://doi. org/10.1161/STROKEAHA.119.025630

42. Schmidbauer MLL (2020) Low rate of intracerebral hemorrhage after cardiac catheterization in patients with acute ischemic stroke in a large case series. Clin Neurol Neurosurg 198:106159. https:// doi.org/10.1016/j.clineuro.2020.106159

Publisher's note Springer Nature remains neutral with regard to jurisdictional claims in published maps and institutional affiliations. 